\title{
An Optimization Model for Dynamic Bulk Provisioning in Elastic Optical Networks
}

\author{
Ajmal Muhammad $^{(1)}$, Marija Furdek ${ }^{(2),(3)}$, Paolo Monti ${ }^{(2)}$, Lena Wosinska ${ }^{(2)}$, Robert \\ Forchheimer $^{(1)}$ \\ (1) Linköping University, Linköping, Sweden, Email: \{ajmal, robert\}@isy.liu.se \\ (2) KTH Royal Institute of Technology, Stockholm, Sweden, Email: \{paolo, wosinska\}@kth.se \\ (3) Dept. of Telecommunications, University of Zagreb, Croatia, Email: marija.furdek@fer.hr
}

\begin{abstract}
We investigate benefits of setup-delay tolerance in elastic optical networks and propose an optimization model for dynamic and concurrent connection provisioning. Simulation shows that the proposed strategy offers significant improvement of the network blocking performance.

(C) 2014 Optical Society of America

OCIS codes: (060.0060 ) Fiber optics and optical communications; (060.4256) Networks, network optimization;
\end{abstract}

\section{Introduction}

Elastic optical networks (EONs), also known as flexgrid networks, are considered as a promising solution able to cope in an efficient way with the diverse bandwidth (i.e., spectrum) requirements of the latest emerging applications. In the EONs paradigm the frequency spectrum is divided into a number of small spectrum slices that are allocated to match as close as possible the spectrum requirement of each connection request. As a result EONs are able to show better spectrum utilization performance when compared to conventional wavelength division multiplexed (WDM) networks based on a coarse and rigid spectral grid [1]. However, in a dynamic provisioning environment, settingup and tearing down connections with different spectrum requirements can lead to the fragmentation of spectrum resources where scattered gaps in the spectrum can reduce the potential spectral efficiency gain of the EON concept. Numerous strategies have been proposed in the literature to address this problem. The fragmentation-aware routing and/or spectrum assignment techniques proposed in [1-3] aim at mitigating the spectrum fragmentation to lower the number of blocked connection requests ( i.e., blocking probability), while the studies in [4-6] present rerouting and spectrum reallocation approaches to be used when the fragmentation and/or the connection request blocking level in the network exceed a certain threshold.

Several network applications, such as grid service requests, might have flexible set-up times for the resource provisioning phase. For such applications, centralized provisioning strategies based on the path computation element (PCE) concept has been proven to be very effective in terms of lowering the connection request blocking level $[7,8]$. In fact, leveraging on their flexible set-up time it is possible for PCE to concurrently process connection requests in bulks (i.e., batches), thus achieving better resource utilization level then provisioning strategies based on the sequential processing of individual requests. The study in this paper aims at investigating the benefits of setup-delay tolerance in elastic optical networks allowing for dynamic bulk provisioning [8], where connection requests are processed in a combined manner with the objective of minimizing the connection request blocking and the fragmentation level in the network. To this end, the elastic bulk provisioning (EBP) problem is formalized as an integer linear program (ILP). A customeroriented service level agreement (SLA) metric, namely, set-up delay tolerance $\left(t_{d}\right)$ [9] is employed as a time threshold parameter for collecting and creating bulks of connection requests. $t_{d}$ describes the maximum time a connection request is willing to wait until resources are provisioned. Connection requests arriving in the network are accumulated for concurrent optimization until the $t_{d}$ of the most impatient request expires. The bulk of requests is then fed to the ILP solver for routing and spectrum assignment. Simulation results show that concurrent processing is also beneficial in EON, showing tangible improvements in the connection request blocking and in the network fragmentation level.

\section{Elastic Bulk Provisioning (EBP) Problem Formulation}

The objective of the elastic bulk provisioning (EBP) problem is to minimize the amount of rejected bandwidth (i.e., total number of spectrum slices not provisioned vs. the total number of spectrum slices requested) while reducing the network fragmentation level. The EBP problem can be modeled as an ILP formulation as follows.

Input parameters:

$\mathscr{G}(\mathscr{V}, \mathscr{E}):$ a directed graph where $\mathscr{V}$ is a set of vertices representing the network nodes and $\mathscr{E}$ a set of arcs representing

This work is supported by the EC FP7, grant agreement No. 318137 DISCUS. 
network fiber links; $\mathrm{F}=\left\{f_{1}, f_{2}, \ldots, f_{|\mathrm{F}|}\right\}$ : set of spectrum slices supported on each fiber link; D: bundle of connection requests to be established in the network; $\Omega_{d}$ : number of slices demanded by request $d \in \mathrm{D} ; \pi^{d}$ : set of candidate paths for $d$; $H_{\pi}$ : set of finer links included in the path $\pi$; $\Upsilon=\left\{K_{1}, K_{2}, \ldots, K_{\left|\cup_{d \in \mathrm{D}} \pi^{d}\right|}\right\}$ : set of constants with distinct values, used for assigning a unique identifier to each path for $\mathrm{D} ; \mathrm{N}_{\pi, f_{s}}$ : number of contiguous and continuous spectrum slices $\left(\Omega_{d} \leq \mathrm{N}_{\pi, f_{\bar{s}}}\right)$ available on path $\pi$, beginning with slice $f_{\bar{s}} ; \Psi_{\pi, f_{\bar{s}}}=\left\{f_{\bar{s}}, f_{\bar{s}+1}, \ldots, f_{\bar{s}+\left(\mathrm{N}_{\pi, f_{\bar{s}}}-\Omega_{d}\right)}\right\}$ : set of candidates for the beginning slice assigned to $d$ on $\mathrm{N}_{\pi, f_{\bar{s}}}$;

\section{Variables:}

$A_{d} \in\{0,1\}$ - equal to 1 if request $d \in \mathrm{D}$ is rejected and 0 otherwise;

$x_{\hat{\pi}^{d}} \in\{0,1\}$ - equal to 1 if path $\hat{\pi}^{d} \in \pi^{d}$ is utilized by $d \in \mathrm{D}$ and 0 otherwise;

$S_{\hat{\pi}^{d}, f_{\hat{s}}} \in\{0,1\}$ - equal to 1 if spectrum segment on path $\hat{\pi}^{d}$ beginning with slice $f_{\hat{s}}$ of the contiguous and continuous slices $\mathrm{N}_{\hat{\pi}^{d}, f_{\bar{s}}}$ is utilized by $d \in \mathrm{D}$, and 0 otherwise;

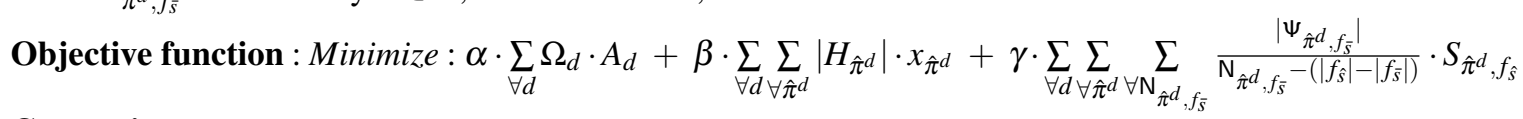

Constraints

\begin{tabular}{|c|c|}
\hline$\sum_{\forall \hat{\pi}^{d}} x_{\hat{\pi}^{d}}+A_{d}=1 \quad \forall d \in \mathrm{D}$ & $\sum_{\forall \hat{\pi}^{d}} K_{\left|\hat{\pi}^{d}\right|} \cdot x_{\hat{\pi}^{d}}-\underset{\forall N_{\hat{\pi}^{d}, f_{\hat{s}}}}{ } K_{\left|\hat{\pi}^{d}\right|} \cdot S_{\hat{\pi}^{d}, f_{\hat{s}}}=0 \quad \forall d \in \mathrm{D}$ \\
\hline 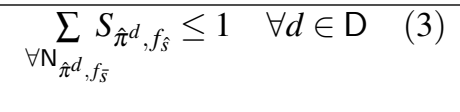 & $S_{\hat{\pi}^{d}, f_{\hat{s}}}+\sum_{\substack{\forall \hat{d} \\
\hat{d} \neq d}} \sum_{\substack{\forall \hat{\pi}^{\hat{d}} \\
\hat{\pi}^{\hat{d}} \cap \hat{\pi}^{d} \neq\left\{\emptyset f_{\hat{s}} \in\left\{\left\{f_{\hat{s}}, f_{\hat{s}+1}, \ldots, f_{\hat{s}+\Omega_{d}}\right\}\right.\right.}} S_{\hat{\pi}^{\hat{d}}, f_{\hat{s}}} \leq 1 \quad \forall f_{\hat{s}} \in \Psi_{\hat{\pi}^{d}, f_{\bar{s}}}, \forall \hat{\pi}^{d} \in \pi^{d}, \forall d \in \mathrm{D}$ \\
\hline
\end{tabular}

Objective function (1) represents a weighted sum where the choice of $\alpha \gg \beta>\gamma$ allows to prioritize the minimization of the first term (i.e., the total bandwidth of all blocked requests). The second term in (1) tries to reduce resource utilization by selecting the shortest path with available spectrum resources. Finally, the third term attempts to lower the spectrum fragmentation by giving preference to slices with a $\mathrm{N}_{\hat{\pi}^{d}, f_{\bar{s}}}$ value exactly equal to $\Omega_{d}$, or, if such spectrum slice does not exist, to the first $\Omega_{d}$ slices on the largest available block, i.e, block with the largest $\mathrm{N}_{\hat{\pi}^{d}, f_{\bar{s}}}$ value. This spectrum allocation policy, called Exact Fit, has been shown to have good blocking probability performance [3]. Constraint (2) ensures that connection requests are either rejected or provisioned on one of the candidate paths. Constraint (3) selects spectrum slices for each connection request. Constraint (4) ensures that the selected spectrum resources are available on the chosen path. Constraint (5) makes sure that each slice is allocated to at most one connection request.

\section{Numerical Results}

The performance of the proposed EBP strategy is evaluated using a custom-built event-driven simulator. Simulations are carried out on the NSF topology [8] with 14 nodes and 42 bidirectional fiber links, each one supporting 80 spectrum slices. Connection requests are assumed to arrive in the network following a Poisson process, each one requiring a number of slices uniformly chosen among $\{2,4,6,8\}$ with an exponentially distributed duration, whose average value is set to 1 time unit. Note that the guardband between allocated spectrum slices is not taken into account, but they can be easily included by modifying constraint (5) accordingly. Source/destination pairs of the connection requests are assumed to be uniformly distributed among all nodes. The number of candidate paths for each connection request is set to 5, while the ILP formulation is solved by running a commercially available ILP solver (CPLEX). The values of $\alpha$ and $\beta$ are experimentally set to 1 and 0.03 , respectively. The value of $\gamma$ is tuned to investigate the effect of different spectrum selection policies on the objective function. The value of $t_{d}$ for each request is set to $5 \%$ or $10 \%$ of the connection holding-time $\left(t_{h}\right)$.

The performance of the EBP approach is assessed in terms of bandwidth blocking ratio (BBR), defined as the ratio between total blocked and requested bandwidth, and of network utilization entropy [10], which measures the level of resource fragmentation. The network utilization entropy is defined as the sum of variations in the usage status of all pairs of neighboring slices on every link divided by the product between the total number of fiber links in the network and the number of available slices per link reduced by 1 . A lower utilization entropy indicates that the resources are utilized in a more ordered manner, with fewer gaps in the slice usage map and a lower level of fragmentation. Simulation results are compared against a baseline (BL) approach that employs the same objective function, but provisions connection requests one by one as they arrive in the network.

The average value of BBR as a function of the load is evaluated in Fig. 1. Three insights can be gained from these results. First, allowing larger values of the time-threshold parameter (i.e., 10\% of $t_{h}$ ) can augment the size of the bundle of connection requests for concurrent optimization, and consequently enhance the network BBR performance. Second, concurrent optimization of connection requests considering only resource utilization (i.e., for $\gamma=0$ ) still has the 


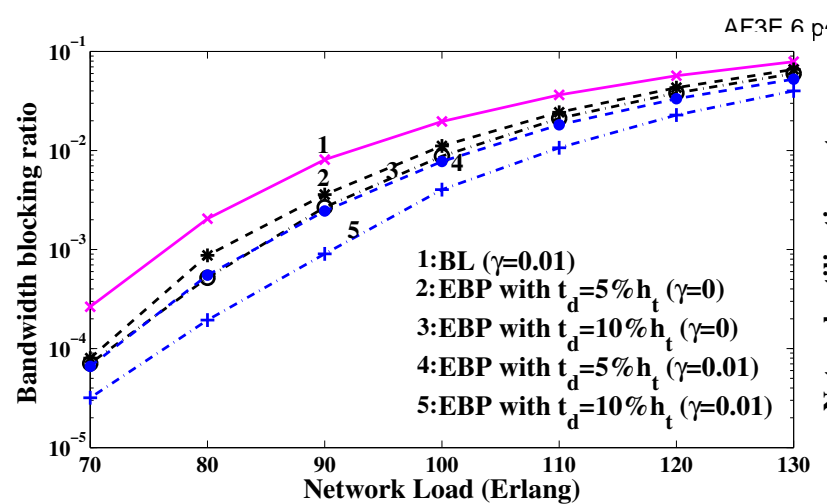

Figure 1: Bandwidth blocking ratio vs. offered network load
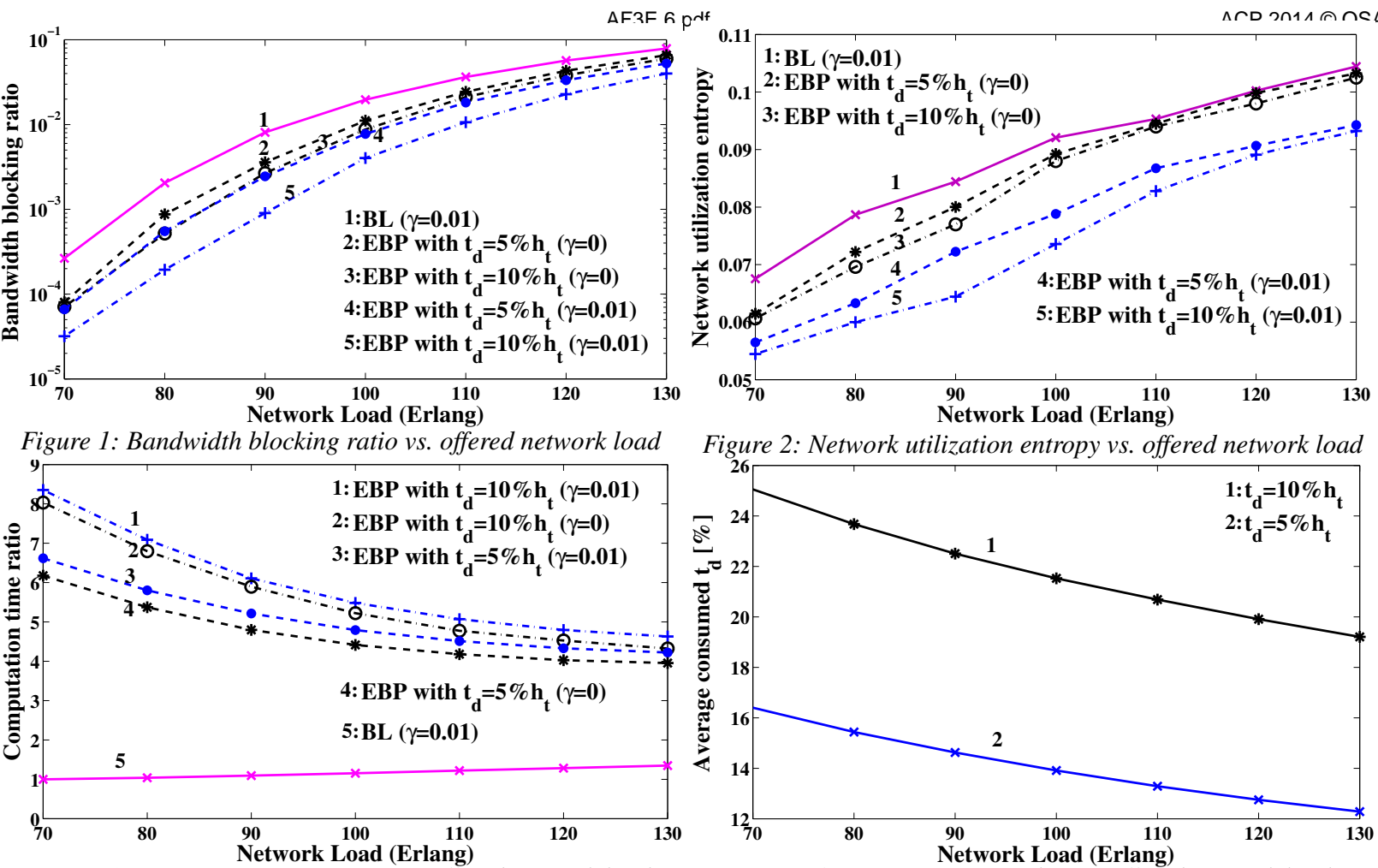

Figure 3: Computation time ratio vs. offered network load

Figure 4: Average consumed $t_{d}$ vs. offered network load

potential to improve BBR compared to sequential provisioning (i.e., BL) which considers both resource and fragmentation minimization. Third, bulk provisioning which additionally minimizes fragmentation (i.e., for $\gamma=0.01$ ), can bring further and significant reductions of the BBR. This indicates that maintaining a low level of fragmentation decreases the chance of rejection for incoming/future connection requests, which in turn leads to an overall improvement in BBR. This latter aspect is further verified by the network utilization entropy values shown in Fig. 2, with the EBP with $\gamma=0.01$ outperforming all other approaches.

In order to examine the feasibility of the proposed model in terms of network scalability, Fig. 3 shows the ratio of computational time for different cases of EBP compared to BL with $\gamma=0.01$. The figure reveals a modest growth (i.e., up to a factor of 8.2) in the computational time for EBP. However, the computational time ratio drops at higher loads owing to a diminished search space for computing the optimal solution. Finally, Fig. 4 exhibits the average percent of $t_{d}$ actually used by each connection request while waiting for provisioning. Due to the fact that the upper bound on the waiting time for all the request in the bundle is set by the most impatient one, the maximum allowed $t_{d}$ is only fractionally exhausted before the bundle is processed for concurrent optimization.

\section{Conclusions}

The paper presents an ILP model for dynamic bulk provisioning of connection requests in elastic optical networks. The proposed model exploits the set-up delay tolerance to bundle the connection requests for concurrent processing with the aim of optimizing resource consumption and (proactively) reducing spectrum fragmentation in the network. Simulation results show that the proposed approach reduces bandwidth blocking ratio by more than $40 \%$ with a modest increase in computational time.

\section{References}

1. Y. Sone et al., "Routing and spectrum assignment algorithm maximizes spectrum utilization in optical networks," ECOC, Mo1K.3, 2011.

2. Y. Yin et al., "Fragmentation-aware routing, modulation and spectrum assignment algorithms in elastic optical...," OFC, OW3A.5, 2012.

3. A. Rosa et al., "Spectrum allocation policy modeling for elastic optical networks," HONET, 2012.

4. A. Casteo et al., "Dynamic routing and spectrum (re)allocation in future flexgrid optical networks," Computer Networks, vol.56, 2012.

5. F. Cugini et al., "Push-pull defragmentation without traffic disruption in flexible grid optical networks," IEEE JLT, vol.56, no.1, 2013.

6. M. Zhang et al., "Spectrum defragmentation algorithms for elastic optical networks using hitless spectrum...," OFC, OW3A.4, 2013.

7. L. Valcarenghi et al., "Experimental evaluation of PCE-based batch provisioning of grid service interconnections," Globecom, 2009.

8. J. Ahmed et al., "A dynamic bulk provisioning framework for concurrent optimization in PCE-based...," IEEE JLT, vol. 30, no.14, 2012.

9. C. Cavdar et al., "Shared-path protection with delay tolerance (SDT) in optical WDM mesh networks," IEEE JLT, vol. 28, no.14, 2010.

10. X. Wang et al., "Utilization entropy for assessing resource fragmentation in optical networks," OFC, OTh1A.2, 2012. 